\title{
中国心理学前沿
}

\section{基于多人脑电一致性特征的 情绪识别探索}

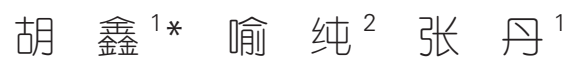

1. 清华大学社会科学学院心理学系, 北京, 100084;

2. 清华大学计算机科学与技术系, 北京, 100084

邮箱: hu-x18@mails.tsinghua.edu.cn

摘 要: 寻找不同情绪状态的脑神经表征, 对于情绪的认知神经机制研究与情 绪识别等现实应用具有重要意义。现有基于脑电的情绪识别研究方法大多采用 经典的事件相关分析方法, 难以应用于包含连续视觉一听觉信息的自然情绪刺 激情境。本研究探索了多人脑电一致性特征用于情绪识别的效力。多人脑电一 致性特征关注多人在同一复杂视听刺激环境下的脑电响应时间过程的相似性, 从有别于事件相关方法的多人视角刻画了大脑神经响应特性。研究发现, 32 名 被试观看 40 段情绪诱发视频时, 被试的左侧顶叶及前额叶脑电响应的多人一 致性特征分别与主观情绪评分的唤起度和效价有显著相关（唤起度 $r=0.41$, $p=0.008$; 效价 $r=0.37, p=0.017$ ) 。进一步研究发现, 基于多人脑电一致性特 征对情绪唤起度和效价主观评分高/低的二分类所得正确率分别为 $77.5 \%$ 和 $70.0 \%$ ，而基于相应的单人脑电特征所得分类正确率仅为随机水平（ $44.1 \pm 13.9 \%$ 和 $48.6 \pm 12.7 \%$ ）。本研究结果首次展示了多人脑电一致性特征在情绪识别领域

文章引用: 胡共, 喻纯, 张丹. 基于多人脑电一致性特征的情绪识别探索 [ J ]. 中国心理学前沿, 2019, 1 ( 3 ): 
的效力与应用潜力。

关键词: 脑电; 多人一致性; 情绪; 分类

收稿日期：2018-08-31; 录用日期：2018-12-29; 发表日期：2019-05-30

\title{
Exploring Emotion Recognition Using EEG-based Inter- subject Correlation Features
}

\author{
Xin $\mathrm{Hu}^{1} *$ Chun $\mathrm{Yu}^{2}$ Dan Zhang ${ }^{1}$
}

1.Department of Psychology, Tsinghua University, Beijing 100084;

2.Department of Computer Science and Technology, Tsinghua University, Beijing

100084

Abstract: Human emotion plays an important role in our daily life. EEG-based emotion recognition is expected to facilitate our understanding of the neural mechanisms as well as applications of emotion recognition. Most EEG-based emotion recognition studies have employed the classical event-related approach, which may not be the most suitable tool for naturalistic situations with continuous audio-visual emotional information streams.In the present study, we explored emotion recognition using EEG-based inter-subject correlation (ISC) features. ISC measures the consistency of neural responses across a group of participants exposed to identical complex and continuous stimuli, characterizing the neural responses from a multi-person perspective. Using a publicly available EEG-based emotion database named DEAP, in which 32 participants watched 40 video clips with different emotional properties, ISCs over left parietal cortex and frontal region are found to be significantly correlated with arousal ratings and valence ratings, respectively (arousal: $r=0.41, p=0.008$; valence: 
$r=0.37, p=0.017)$. In addition, using ISCs as features, binary classification accuracies for arousal and valence reached $77.5 \%$ and $70 \%$, which are superior to the traditional individual spectral power based method $(44.1 \pm 13.9 \%$ and $48.6 \pm 12.7 \%)$. Our results suggest that the inter-subject correlation approach as an effective and promising candidate for investigating human emotion experiences.

Key words: EEG; Inter-subject correlation; Emotion; Classification

Received: 2018-08-31; Accepted; 2018-12-29; Published; 2019-05-30

Copyright ( $\odot 2019$ by author(s) and SciScan Publishing Limited.

This article is licensed under a Creative Commons Attribution-NonCommercial 4.0 International License.

https://creativecommons.org/licenses/by-nc/4.0/

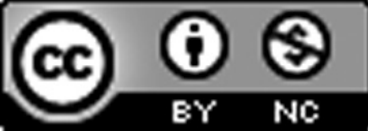

情绪是人类在认知和意识过程中，综合多种感觉、思想和行为产生的心理 和生理状态，在人们的日常生活中有着非常重要的作用。大脑作为人类思维活 动的中枢，是情绪信息处理的物质基础。因此，对情绪体验过程中的大脑神经 信号进行建模分析，是最直接的情绪识别方法。相比主观报告或面部表情、语 音语调、身体姿态等行为指标，基于大脑神经信号的情绪识别具有客观、不易 伪装等特点，在理论上可以取得更准确、可靠的识别结果 $[1]$ [2]。在神经 机制研究的层面，基于大脑神经信号的有效情绪识别有助于发现不同情绪状态 对应的脑神经响应模式，为情绪的脑神经机制提供新的研究思路与参考信息。

有效的情绪诱发是有效情绪识别的重要基础。常用的情绪诱发方法包括 Velten 情绪诱发法（引导被试阅读具有强烈情绪色彩的语句并体验语句表达的 情绪）、视频诱发法、音乐诱发法、图片诱发法、想象诱发法等。在基于神经 响应的事件相关分析方法 (如事件相关电位分析，ERP）的情绪研究中，由于实 
验范式的限制，通常采用快速呈现的情绪图片、词汇或声音来诱发被试的情绪 状态。这一范式下的常用诱发材料包括 Lang 等人（1997）开发的国际情绪图片 系统（International Affective Picture System，IAPS ）、Brandley 和 Lang（1999）开 发的英语情感词系统（Affective Norms for English Words，ANEW），以及 Lang、 Bradley 和 Cuthbert ( 1999 ) 开发的国际情感数码声音系统（International Affective Digitized Sounds, IADS ) 等，但是此类短时程的单通道情绪诱发材料通常与真实 生活中的情绪体验存在很大的差异。有元分析对不同情绪诱发手段的诱发有效 性进行了比较，发现同时包含视觉与听觉刺激的视频诱发方法比其他常见的情 绪诱发方式具有更好的生态效度和情绪诱发效果 [3]。近年来的一些研究开始 关注用视频作为诱发手段时的情绪神经响应建模与识别 [4]［5］［6]。视频 诱发材料具有连续的复杂视听觉信息，有更高的生态效度，更加接近自然情绪 刺激情境。然而，视频诱发材料往往包含较长时间（几十秒或更长）的复杂视 觉和听觉信息，不利于进行事件编码，使得传统的事件相关分析方法不再适用。

针对以视频为代表的更加接近自然情境的长时程诱发材料, 近年来社会神 经科学领域学者提出多人神经响应一致性（Inter-Subject Correlation, ISC）分析 方法 [7]，从不同人在接受同样外部环境感知觉信息时的神经响应在时间、空 间等维度上的相似性的角度来研究大脑认知神经活动规律。基于功能磁共振成 像技术与脑电技术的多人神经响应一致性分析已经应用于记忆 $[8]$ ［13］、言 语交互 $[14 ］$ ［15］［16］、视觉信息加工 ～7 ］、音乐信息加工 ～17］［18］、 儿童发展 [19］、自闭症 $[21]$ 以及抑有症 $[10]$ [20 ] 等相关研究中，并取得 了一系列有别于传统事件相关研究的全新角度的神经科学发现。在情绪体验相 关领域，研究者也已经开展了一些前沿探索，发现 ISC 指标可以反映观众对电 影的喜好倾向 ～9］［12］，且多个情绪相关脑区都观察到显著的 ISC 活动 [ 11 ]。 比如 Nummenmaa 等 (2012) 发现, 消极情绪可以引起情绪加工相关脑区 (丘脑、 腹侧纹状体、内侧前额叶、前扣带回）与默认模式网络（楔前叶、题顶交界区、 腹内侧前额叶）的 ISC 上升；高唤起的情绪则伴随着躯体感觉皮层、视觉皮层、 双侧顶内沟和额眼区的 ISC 上升 [11]。然而, 上述研究主要是通过功能磁共 振成像技术开展，基于脑电技术的研究相对较少。相比功能磁共振技术，脑电 
技术空间精度较低，但时间分辨率更高，可以更好地跟随情绪变化的时间过程。 此外，脑电技术的测量成本更低，应用成熟度也更高。因此，基于脑电技术的 多人神经响应一致性分析（以下简称多人脑电一致性分析）方法在情绪研究上 还有更多的探索空间。特别在情绪研究中，核磁设备的高噪声封闭环境对于被 试的情绪体验（尤其是积极情绪）存在难以避免的干扰，而脑电技术相较之下 能够提供更为自然和舒适的情绪体验环境。

综上所述，本研究选择 Koelstra 等人 2012 年发布的公开情绪生理数据库 DEAP ( Database for Emotion Analysis Using Physiological Signals ) [22] 中的脑电 数据展开分析。考虑到近年来多人脑电一致性分析方法在情绪研究领域初步展 现出潜力, 但是该方法与传统的个体层面分析方法在情绪研究上的分析效力尚 缺乏充分的对比讨论，本研究将同时从个体和群体两个层面开展研究。在个体 层面上，本研究沿用情绪识别领域常用的基于脑电频谱能量特征的分析方法; 在群体层面上，本研究将基于多人脑电一致性分析开展探索，并通过两种方法 取得的情绪分类正确率对其情绪识别效力进行比较。

\section{1 数据概述}

情绪生理数据库 DEAP 主要通过音乐视频材料诱发下产生的多种外周生理信 号与脑电信号，以下简要介绍诱发材料的选取与脑电数据的采集过程。

DEAP 的实验者主要通过三个步骤笁选实验所用的情绪诱发视频：(1) 在知 名音乐视频网站 Last. fm 等处寻找不同类别的音乐视频片段，共计得到 120 个片 段。(2) 将每段音乐视频的声音幅度、音调以及画面的亮度、色彩等特征作为唤 起度和效价的指标，综合两种指标得到情绪高潮指数，并选取音乐视频中情绪 高潮指数最高的 1 分钟片段作为该段音乐视频的最终采用片段。(3) 邀请志愿者 对 120 个 1 分钟音乐视频片段的唤起度与效价等进行主观评分，每段音乐视频 获得不少于 14 人的评分数据，选择其中平均评分最高并且评分方差最小的 40 段音乐视频作为最终的实验材料。

在正式实验阶段，选取 32 位健康被试（男女各半，年龄在 $19 \sim 37$ 岁，平 均年龄为 26.9 岁）观看 40 段具有不同情绪属性的音乐视频片段，观影过程中的 
脑电通过 32 导主动式电极脑电设备 (BioSemi, 荷兰) 进行记录, 采样率设定为 $512 \mathrm{~Hz}$ 。观看完每段视频之后，被试需要在 $1 \sim 9$ 分的连续量表上对当前自我的 情绪状态进行评估，包括唤起度、效价、喜爱度与主控感四个评分维度。其中， 唤起度与效价是经典的情绪二维模型中的核心维度 [23]，本研究的数据分析 将主要围绕这两个维度展开。具体实验流程如图 1 所示。

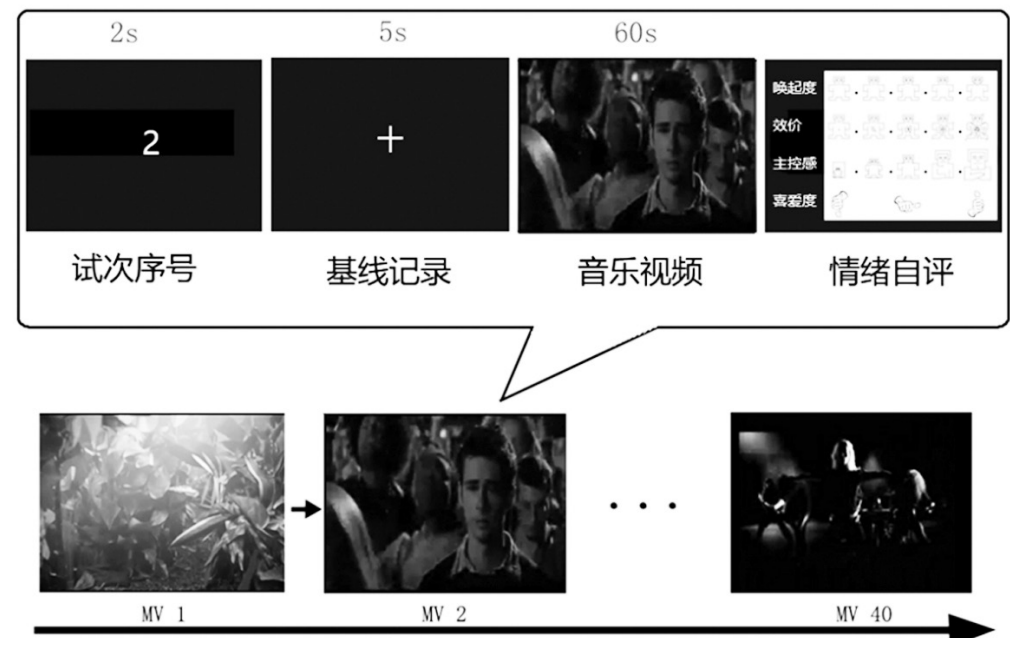

图 1 DEAP 实验流程示意

注: 被试需观看 40 段情绪诱发视频, 观看每段视频前, 首先呈现 2 秒试次序号, 然后 呈现 5 秒注视点作为基线记录，接着呈现 60 秒音乐视频，最后在每段视频结束后，被试对视 频的唤起度、效价、主控感与喜爱度进行评分。

\section{2 分析与结果}

首先将脑电信号降采样到 $256 \mathrm{~Hz}$ ，并进行 $0.05 \sim 50 \mathrm{~Hz}$ 滤波。然后采用独 立成分分析的方法将眼动成分剔除，得到去除眼动伪迹的脑电信号。在特征提 取的过程中, 出于最大化情绪强度的考虑 [22], 截取每段视频的最后 30 秒数 据进行后续分析，并以每段视频开始播放前 5 秒作为基线进行基线校正。

对于脑电特征的提取, 首先将脑电信号滤波至 theta $(3 \sim 7 \mathrm{~Hz})$ 、alpha $(8 \sim 13$ $\mathrm{Hz})$ 、 beta $(14 \sim 29 \mathrm{~Hz})$ 与 gamma $(30 \sim 47 \mathrm{~Hz})$ 频带, 然后并行提取个体 层面和群体层面的脑电特征。在个体层面上，在滤波后信号的基础上计算每段 
视频后 30 秒的脑电在上述频带的信号能量，每位被试均取得 128 维度个体脑电 能量特征（32 通道 $\times 4$ 频带能量）。在群体层面上, 分别计算各频带信号时域 波形的多人一致性（ISC）指标，得到 128 维度群体 ISC 特征（32 通道 $\times 4$ 频带 ISC 指标）。ISC 的具体计算方法如下：对所有 32 名被试，分别计算所有两两配 对 (共 496 个配对) 的脑电信号的皮尔逊相关系数，并选取所有配对的相关系数 均值作为 ISC 指标。

$$
\mathrm{ISC}=\frac{1}{\frac{m(m-1)}{2}} \sum_{i=1}^{m} \sum_{j=2, j>i}^{m} r_{i j}
$$

得到两类脑电特征之后，分别计算这两种脑电特征各维度与被试主观情绪 评分的皮尔逊相关系数。其中，脑电能量特征与情绪评分的相关系数在个体层 面展开计算，即对每名被试，计算 40 段视频的脑电能量特征与对应情绪评分的 相关系数, 然后计算所有被试相关系数的均值; ISC 与主观评分的相关系数在群 体层面展开计算，即对每段视频，计算被试间平均情绪评分，然后计算 40 段视 频的 ISC 特征与对应平均视频情绪评分的相关系数, 结果分别如图 2 与图 3 所示。

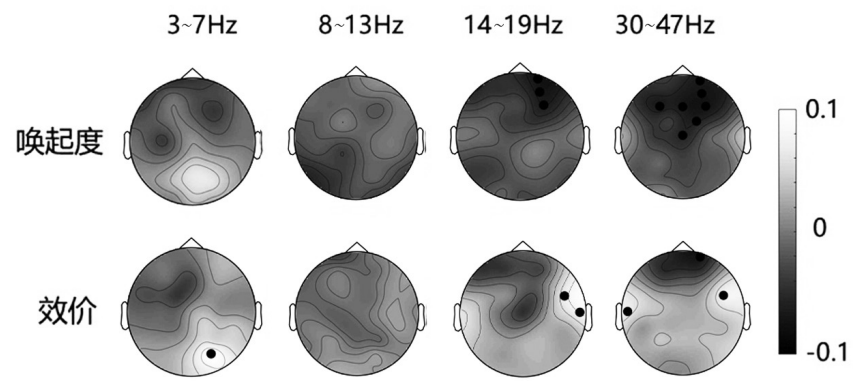

图 2 个体情绪评分与个体脑电能量特征平均相关系数

注: 黑点标注的通道表示该特征与情绪评分显著相关 $(p<0.05, p$ 值使用 FDR 方法进 行了多重比较校正）（Genovese，Lazar，\& Nichols，2002）。 


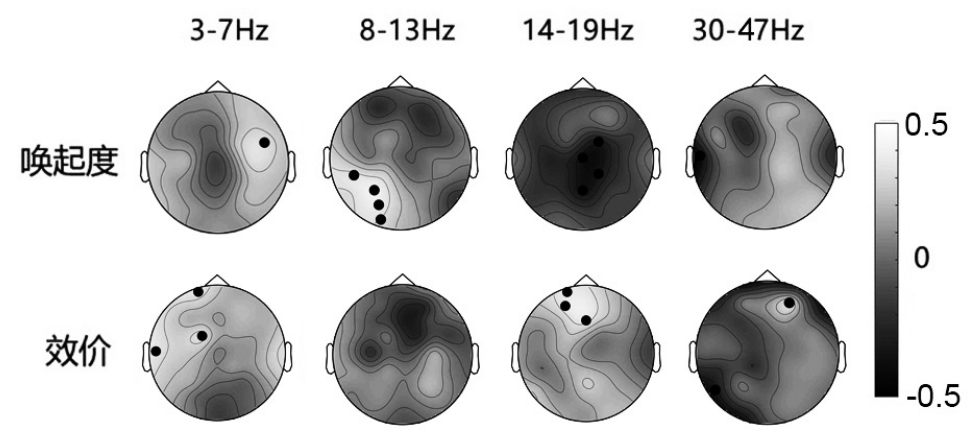

图 3 群体情绪评分与多人脑电一致性特征 (ISC) 相关系数

注: 黑点标注的通道表示该特征与情绪评分显著相关 $(p<0.05, p$ 值使用 FDR 方法进 行了多重比较校正）。

对比两项结果发现，个体脑电能量与多人脑电一致性特征对情绪体验有 不同的表征模式。个体脑电能量特征与情绪唤起度的最强相关是右侧额区的 gamma 频带能量 $(r=-0.07, p=0.02)$ ，与情绪效价最强的相关为右侧顶枕区 theta 频带能量（ $r=0.10, p<0.001)$ （见图 2 ）。多人脑电一致性特征与情绪唤 起度相关最强的脑电特征是左侧顶区 alpha 频带的 ISC $(r=0.41, p=0.008)$ （见 图 4 ); 与情绪效价相关最强的脑电特征则是前额区域 beta 频带的 ISC $(r=0.37$, $p=0.017$ ）（见图 5 ）。

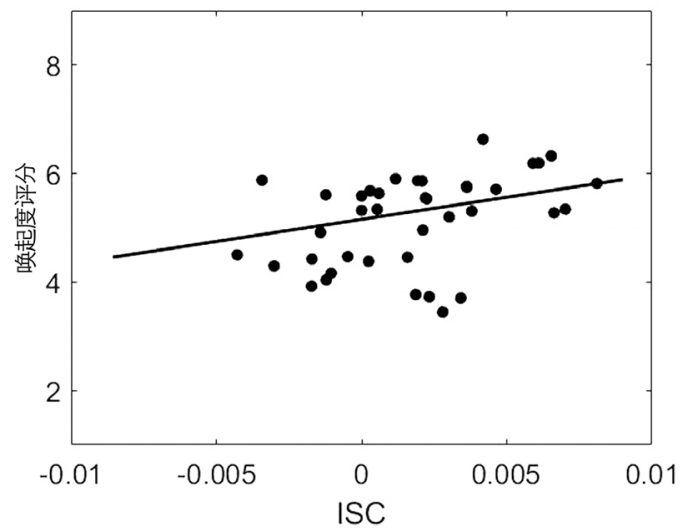

图 4 每段音乐视频的 ISC 指标与唤起度平均得分 ( $8 \sim 13 \mathrm{~Hz}$, P3 电极 ) 


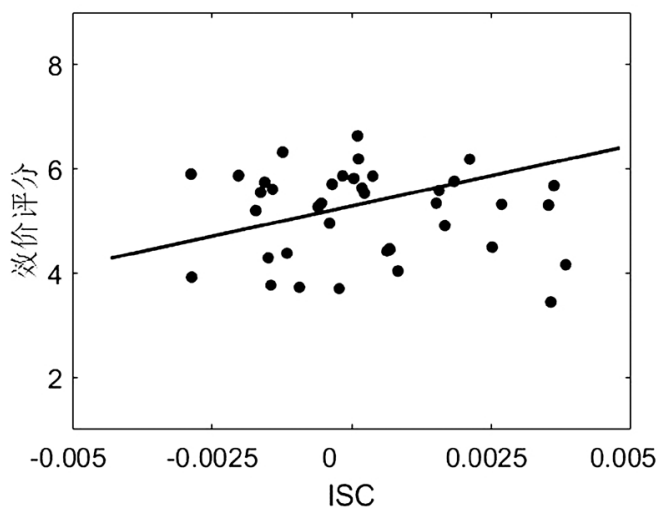

图 5 每段音乐视频的 ISC 指标与效价平均得分（14 $29 \mathrm{~Hz}, A F 3$ 电极）

为了进一步探索个体脑电能量特征与多人脑电一致性特征对情绪体验的表 征效力, 本研究分别利用两类脑电特征构建支持向量机（SVM）分类器，从效 价和唤起度两个维度对 40 段音乐视频进行分类。具体来说，根据 40 段音乐视 频在情绪维度上的得分将其分为高 / 低分组，并赋予相应的标签，并以全部 32 通道在 4 个脑电频带上的脑电特征（共计 128 维特征）进行分类。基于多频带 个体能量特征的分类器获得的十折交叉检验的平均正确率分别为 $44.1 \% \pm 13.9 \%$ （唤起度维度）和 $48.6 \% \pm 12.7 \%$ （效价维度）；而基于多频带 ISC 特征的分类 器获得的十折交叉检验的正确率分别为 $77.5 \%$ （唤起度维度）和 70.0\%（效价维 度），均显著高于基于个体脑电能量的分类正确率（ $p<0.001 ） 。$ 详细结果如 表 1 所示。

表 1 基于 ISC 特征与个体脑电能量特征的情绪二分类正确率

\begin{tabular}{|c|c|c|c|c|c|}
\hline \multicolumn{6}{|c|}{ 基于 ISC 特征的分类正确率 } \\
\hline & $3 \sim 7 \mathrm{~Hz}$ & $8 \sim 13 \mathrm{~Hz}$ & $14 \sim 29 \mathrm{~Hz}$ & $30 \sim 47 \mathrm{~Hz}$ & 所有频带 \\
\hline 唤起度 & $57.5 \%$ & $82.5 \%$ & $37.5 \%$ & $47.5 \%$ & $77.5 \%$ \\
\hline 效价 & $22.5 \%$ & $37.5 \%$ & $42.5 \%$ & $27.5 \%$ & $70.0 \%$ \\
\hline \multicolumn{6}{|c|}{ 基于个体脑电能量特征的分类正确率 $( \pm$ 标准差 $)$} \\
\hline & $3 \sim 7 \mathrm{~Hz}$ & $8 \sim 13 \mathrm{~Hz}$ & $14 \sim 29 \mathrm{~Hz}$ & $30 \sim 47 \mathrm{~Hz}$ & 所有频带 \\
\hline 唤起度 & $48.6 \% \pm 15.4 \%$ & $43.4 \% \pm 15.6 \%$ & $45.2 \% \pm 16.2 \%$ & $43.4 \% \pm 13.7 \%$ & $44.1 \% \pm 13.9 \%$ \\
\hline 效价 & $42.1 \% \pm 15.9 \%$ & $44.1 \% \pm 13.2 \%$ & $42.8 \% \pm 18.4 \%$ & $40 \% \pm 16.4 \%$ & $48.6 \% \pm 12.7 \%$ \\
\hline
\end{tabular}

注: 所有频带指结合 $3 \sim 7 \mathrm{~Hz}, 8 \sim 13 \mathrm{~Hz}, 14 \sim 29 \mathrm{~Hz}, 30 \sim 47 \mathrm{~Hz}$ 频带能量作为特 征进行分类。 
本研究进一步比较了基于每位被试个体脑电能量的情绪分类器与基于多 人脑电一致性特征的情绪分类器在唤起度与效价两个维度上的分类正确率。 两类分类器的特征均包含所有频带的脑电特征 $(4$ 频带 $\times 32$ 通道 $=128$ 维特 征）。对比发现，不同被试的个体情绪分类器表现出较大的个体间差异（唤 起度正确率标准差为 $13.9 \%$ ，效价正确率标准差为 $12.7 \%$ ），且部分被试个 体内的情绪唤起度与效价分类正确率差异也较大，比如效价维度的最佳被 试 ( $75.0 \%$ ) 在唤起度上的分类正确率仅为 $32.5 \%$; 唤起度维度的最佳被试 (80.0\%) 在效价上的分类正确率仅为 $35.0 \%$ 。对于每位被试，分别计算其在 唤起度与效价维度的分类正确率均值，发现 32 位被试在唤起度与效价维度的 分类正确率均值在 $31.3 \%$ 到 $65.0 \%$ 之间（平均值为 $46.4 \%$, 标准差为 $8.4 \%$ )。 而基于多人脑电一致性的情绪分类器在唤起度与效价上分别取得了 $77.5 \%$ 与 $70.0 \%$ 的正确率。

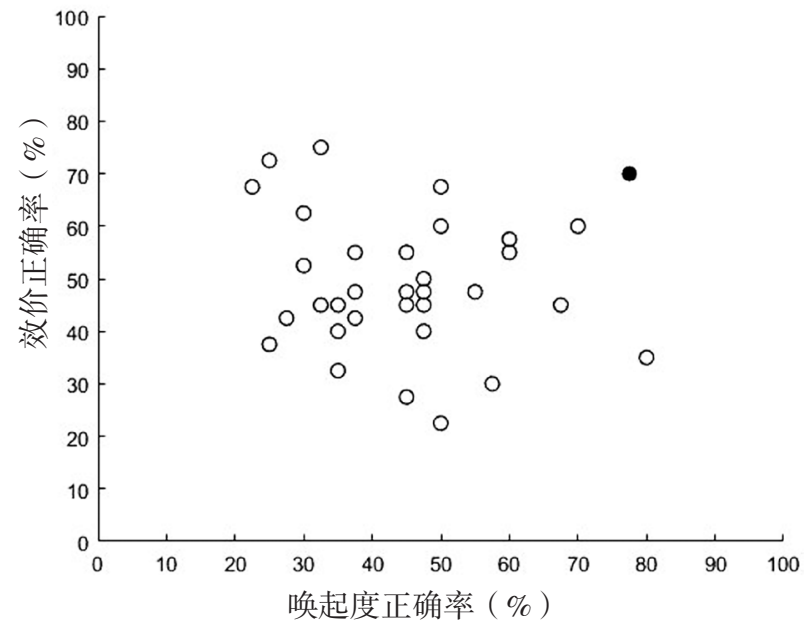

图 6 个体脑电能量情绪分类器与多人脑电一致性情绪分类器的正确率对比

图中白点表示每位被试的个体情绪分类器在唤起度与效价维度的分类正确 率，黑点表示多人脑电一致性情绪分类器在唤起度与效价维度的分类正确率。

\section{3 讨论}

相关性分析结果表明，多人脑电一致性特征与个体脑电能量特征对于情绪 体验具有不同的表征模式，这也意味着多人脑电一致性特征可以提供有别于传 
统方法的全新维度信息。但需要指出的是, 个体脑电能量分析方法与多人脑电 一致性分析方法的分析层次并不一样：个体脑电能量分析是基于个体为单位的 脑电特征，多人脑电一致性分析则是基于群体为单位的脑电特征，关注个体间 的神经活动的共性。

情绪识别分析发现，多人脑电一致性特征比个体脑电能量特征表现出对于 情绪体验的更佳预测力（唤起度预测正确率提升 $33.4 \%$, 效价预测正确率提升 $21.4 \%$ ）。而个体脑电能量特征在情绪识别上的不理想结果可能源自被试间的个 体差异: 本研究在两个情绪维度上都发现了分类正确率超过 $70 \%$ 的 “最佳被试”, 但也存在较多分类正确率并不理想，甚至低于随机水平的被试，导致两个维度 上最终取得的平均正确率仅为 $44.1 \%$ 与 $48.6 \%$; 另一方面, 某一情绪维度上分 类正确率最佳的被试往往不一定能在另一情绪维度也取得理想结果，32 位被试 在情绪唤起度与效价上的平均情绪识别正确率最高为 $65.0 \%$ 。相较之下，基于多 人脑电一致性分析的情绪分类结果在情绪唤起度与效价两个维度均取得了 $70.0 \%$ 及以上的正确率，表现出更为稳健均衡的情绪识别效力。同时，结合多频带多 人脑电一致性特征的情绪分类结果比绝大多数单频带特征表现都出了更高的分 类准确率，表示不同频带的脑电一致性特征具有各自相对独特的贡献，而基于 个体能量特征在不同频带之间的信息冗余度可能较大。

在神经机制的层面，本研究所得个体能量特征与情绪维度的相关性与前人 研究是一致的，如顶枕区 theta 能量上升表达的是愉悦情绪自上而下加工过程伴 随着的视觉皮层相对抑制 [22]，前额区 gamma 能量下降表达的是不同唤起度 下注意和认知功能水平的差异 [24]。多人脑电一致性特征方面，基于功能磁 共振成像多人脑血氧代谢信号一致性响应的研究报告了情绪效价与内侧前额皮 质的关联 [25]，而本研究也观察到情绪效价与前额区域脑电多人一致性特征 的相关性，主要体现在 beta 与 alpha 频带。然而，目前关于多人脑电一致性的 研究还非常有限，不同频带的多人脑电一致性特征的生理机制解释还有待更多 后续研究的探索。

本研究结果有很好的实际应用潜力。虽然基于多人脑电一致性的分析方法 不能针对个体情绪状态进行识别，但基于群体的情绪识别依然有很多现实应用 
需求，比如神经营销领域常关注某部影片或广告会诱发出怎样的普遍性情绪等

[9］［12］［26］。本研究所提出的多人脑电一致性分析方法相比传统个体脑 电能量方法有更好的情绪体验预测力，相关结果有望推进情绪识别应用的进一 步发展，并为情绪的脑神经机制研究提供新的技术方法支持。

\section{基金项目}

国家重点研发计划项目（2016YFB1001200）；清华大学自主科研计划项目 ( 2014Z21043)。

\section{参考文献}

[1] Picard R W, Vyzas E, Healey J. Toward machine emotional intelligence: analysis of affective physiological state [ J ] . IEEE Transactions on Pattern Analysis and Machine Intelligence, 2001, 23 (10): 1175-1191.

https://doi.org/10.1109/34.954607

[2] Frantzidis C A, Papadelis C L, Bamidis P D. Toward emotion aware computing: an integrated approach using multichannel neurophysiological recordings and affective visual stimuli $[\mathrm{J}]$. IEEE Transactions on Information Technology in Biomedicine, 2010, 14 (3): 589-597. https://doi.org/10.1109/TITB.2010.2041553

[ 3 ] Westermann R, Spies K, Stahl G, et al. Relative effectiveness and validity of mood induction procedures: a meta-analysis $[\mathrm{J}]$. European Journal of Social Psychology, 1996, 26 (4): 557-580. https://doi.org/10.1002/ (SICI)1099-0992 (199607)26:4<557::AID-EJSP769>3.0.CO;2-4

[4] Murugappan M, Ramachandran N, Sazali Y. Classification of human emotion from EEG using discrete wavelet transform $[\mathrm{J}]$. Journal of Biomedical Science and Engineering, 2010, 3 (4): 390. https://doi.org/10.4236/jbise.2010.34054

[ 5 ] Soleymani M, Pantic M, Pun T. Multimodal emotion recognition in response to videos [ J ] . IEEE transactions on affective computing, 2012, 3 (2): 211-223. https://doi.org/10.1109/T-AFFC.2011.37 
[6] Castermans T, Duvinage M, Riche N. Online Emotion Classification from Electroencephalographic Signals: A First Study Conducted in a Realistic Movie Theater $[\mathrm{C}]$. International Conference on Artificial Intelligence and Soft Computing. Berlin, Heidelberg: Springer, 2013. https://doi.org/10.1007/978-3-642-38610-7_24

[ 7 ] Hasson, U. Intersubject Synchronization of Cortical Activity During Natural Vision [ J ] . Science, 2004, 303 (5664): 1634-1640. https://doi.org/10.1126/science.1089506

[ 8 ] Hasson U, Furman O, Clark D, et al. Enhanced Intersubject Correlations during Movie Viewing Correlate with Successful Episodic Encoding [ J ] . Neuron, 2008, 57 (3): 452-462. https://doi.org/10.1016/j.neuron.2007.12.009

[ 9 ] Hasson U, Landesman O, Knappmeyer B, et al. Neurocinematics: The neuroscience of films [ J ] . Projections, 2008, 2 (1): 1-26.

https://doi.org/10.3167/proj.2008.020102

[ 10 ] Hasson U, Avidan G, Gelbard H, et al. Shared and idiosyncratic cortical activation patterns in autism revealed under continuous real-life viewing conditions $[\mathrm{J}]$. Autism Research Official Journal of the International Society for Autism Research, 2010, 2 (4): 220-231. https://doi.org/10.1002/aur.89

[11] Nummenmaa L, Glerean E, Viinikainen M, et al. Emotions promote social interaction by synchronizing brain activity across individuals $[\mathrm{J}]$. Proceedings of the National Academy of Sciences, 2012, 109 (24): 9599-9604.

\section{https://doi.org/10.1073/pnas.1206095109}

[12] Dmochowski J P, Bezdek M A, Abelson B P, et al. Audience preferences are predicted by temporal reliability of neural processing $[\mathrm{J}]$. Nature Communications, 2014, 5. https://doi.org/10.1038/ncomms5567

[ 13 ] van Kesteren M T, Fern á ndez G, Norris D G, et al. Persistent schema-dependent hippocampal-neocortical connectivity during memory encoding and postencoding rest in humans $[\mathrm{J}]$. Proceedings of the National Academy of Sciences, 2010, 
107 (16): 7550-7555. https://doi.org/10.1073/pnas.0914892107

[14 ] Wilson S M, Molnar-Szakacs I, Iacoboni M. Beyond Superior Temporal Cortex: Intersubject Correlations in Narrative Speech Comprehension [ J ] . Cerebral Cortex, 2008, 18 (1): 230-242. https://doi.org/10.1093/cercor/bhm049

[ 15 ] Stephens G J, Silbert L J, Hasson U. Speaker-listener neural coupling underlies successful communication [ J ] . Proc Natl Acad Sci U S A, 2010, 107 (32): 14425-14430. https://doi.org/10.1073/pnas.1008662107

[ 16 ] Silbert L J, Honey C J, Simony E, et al. Coupled neural systems underlie the production and comprehension of naturalistic narrative speech $[\mathrm{J}]$. Proceedings of the National Academy of Sciences, 2014, 111 (43): 4687-4696. https://doi.org/10.1073/pnas.1323812111

[17] Abrams D A, Ryali S, Chen T, et al. Inter-subject synchronization of brain responses during natural music listening $[\mathrm{J}]$. European Journal of Neuroscience, 2013, 37 (9): 1458-1469. https://doi.org/10.1111/ejn.12173

[ 18 ] Trost W, Fr ü hholz, Sascha, Cochrane T, et al. Temporal dynamics of musical emotions examined through intersubject synchrony of brain activity $[\mathrm{J}]$. Social Cognitive and Affective Neuroscience, 2015. https://doi.org/10.1093/scan/nsv060

[19] Cantlon J F, Li R, Posner M. Neural Activity during Natural Viewing of Sesame Street Statistically Predicts Test Scores in Early Childhood [ J ] . PLoS Biology, 2013, 11 (1). https://doi.org/10.1371/journal.pbio.1001462

[20 ] Guo C C, Nguyen V T, Hyett M P, et al. Out-of-sync: disrupted neural activity in emotional circuitry during film viewing in melancholic depression $[\mathrm{J}]$. Scientific Reports, 2015, 5: 11605. https://doi.org/10.1038/srep11605

[21 ] Salmi J, Roine U, Glerean E, et al. The brains of high functioning autistic individuals do not synchronize with those of others $[\mathrm{J}]$. NeuroImage: Clinical, 2013, 3: 489-497. https://doi.org/10.1016/j.nicl.2013.10.011

[ 22 ] Koelstra S, Muhl C, Soleymani M, et al. DEAP: A Database for Emotion Analysis; 
Using Physiological Signals [ J ] . IEEE Transactions on Affective Computing, 2012, 3 (1): 18-31. https://doi.org/10.1109/T-AFFC.2011.15

[ 23 ] Russell, James A. Evidence of convergent validity on the dimensions of affect [ J ]. Journal of Personality and Social Psychology, 1978, 36 (10): 1152-1168.

https://doi.org/10.1037//0022-3514.36.10.1152

[24 ] Benasich A A, Gou Z, Choudhury N, et al. Early cognitive and language skills are linked to resting frontal gamma power across the first 3 years $[\mathrm{J}]$. Behavioural Brain Research, 2008, 195 (2): 215-222.

https://doi.org/10.1016/j.bbr.2008.08.049

[ 25] Chavez R S, Heatherton T F. Representational Similarity of Social and Valence Information in the Medial pFC [ J ] . Journal of Cognitive Neuroscience, 2015, 27 (1): 73-82. https://doi.org/10.1162/jocn_a_00697

[26] Dmochowski J P, Paul S, Joao D, et al. Correlated Components of Ongoing EEG Point to Emotionally Laden Attention - A Possible Marker of Engagement? [ J ] . Frontiers in Human Neuroscience, 2012, 6.

https://doi.org/10.3389/fnhum.2012.00112 